a month he is allowed to get about with crutches or in a chair. Such patients will in the course of nature not have very long to live, but their invalidity will be comparatively comfortable.

I think that at the present moment there is some danger of Smith-Petersen's method becoming discredited because it is used for cases in which failure is inevitable.

\section{Treatment of Cases of Non-union}

The existence of non-union unamenable to anything but operative procedures is determined by the time that has elapsed since the accident, and also by the appearances of the fracture surfaces. Usually, if there is no union after two or three months, it will never occur without direct attack. The ends of the bones are rounded off or sclerosed. The choice now lies between four courses: a walking calliper, an operation for uniting the fracture, subtrochanteric osteotomy, and reconstruction of the hip.

As regards the first it may be stated that there is no satisfactory walking splint, and that if nothing can be done these patients will be but poor cripples for the rest of their lives. The reunion of the fracture by open operation should always be the method of choice provided the patient is reasonably healthy and active, and also that there is neither necrosis of the head nor absorption of the neck. I have succeeded in producing a perfect result operating eighteen months after the accident, but the patient was only 31 , was of spare habit and active temperament, and both bones were practically normal. Such a happy result is exceptional ; in the majority of cases of non-union, therefore, we have to choose between osteotomy or a hip reconstruction.

Osteotomy is simple and free from danger, but it involves prolonged plaster fixation and gives very variable results. It is the best that can be done for feeble, elderly patients. The reconstruction operation of Whitman is probably the best treatment for the majority of cases of definite non-union. The head of the bone is removed, the great trochanter transposed to a lower position, and the neck of the femur is thrust into the acetabulum.

In a recent interesting summary of the subject, Kellogg Speed $^{2}$ has termed this " the unsolved fracture," but I think that the Smith-Petersen invention has gone a long way towards its solution, and now what is wanted is to have a systematic report and follow-up of a large number of cases, so that we may be in a position to be sure of the facts both of treatment and of prognosis.

\section{REFERENCES}

${ }^{1}$ Hey Groves: Journ. Bone and Joint Surg., January, 1930, xii, 1. 2 Kellogg Speed: Surg., Gynecol. and Obstet., February 15th, 1935, p. 341 .

The Guide to Current Official Statistics (volume xiii, 1934), which is prepared under the auspices of a Permanent Committee of Departmental Statisticians and is published annually, provides" a comprehensive index to the extensive numerical data published each year in the form of official reports, returns, accounts, and periodicals. Its contents are arranged on a plan which enables the investigator to compile, in the space of a few minutes, a complete list of sources of information on the subject of his inquiry. The degree of analysis of the figures, the date and place to which they relate, and the prices of the volumes in which they are to be found are also indicated. The present volume contains 350 pages and costs $1 \mathrm{~s}$. net, or by post $1 \mathrm{~s}$. 5d. It may be obtained direct from the sale offices of H.M. Stationery Office or through any bookseller. Some of the earlier volumes, dealing with the statistics of previous years, are also available at the same price.

\section{RADIOGRAPHIC DIAGNOSIS IN DISEASES OF THE LUNGS *}

BY

\section{J. G. EDWARDS, M.B., CH.M.SyDNEY}

HONORARY RADIOLOGIST, SYDNEY HOSPITAL; HONORARY CONSULTANT RADIOLOGIST, LEWISHAM HOSPITAL, SYDNEY; MEDICAL AUTHORITY ON RADIOLOGY, N.S.W. SILICOSIS COMMISSION

During my twenty-seven years' experience of radiology I have seen much change in this branch of work, and have known many weird and wonderful types of apparatus, from the induction coil with the dipper break to the large four- and six-valve transformer machines. With the early coil sets it was not possible to take films of the lungs at rest, and fluoroscopic examination was of the greater importance, while in these latter days the film is almost exclusively relied upon for diagnosis.

The short time allowed for this paper does not permit an exhaustive study of this large subject, and I wish to confine my remarks to a few interesting conditions rather than weary you with a set lecture on the more commonplace ones. I will deal shortly with the technique of examination, with various puzzling cases met with in daily life, and with that important condition, silicosis. The changes in the lung from recurrent catarrhal conditions, from tuberculosis, and from malignancy will only receive passing mention.

The value of radiographic examination in chest work is now generally recognized even by the most conservative physicians, and no chest investigation can be considered complete unless a proper radiographic examination has been made. By proper radiographic examination is meant one that has been carried out by a specially trained medical expert; the physician who attempts to interpret his own films is not getting full value from the method. Physician and radiologist should meet after making their individual examinations, and then correlate their findings ; if the physician is aware of the radiologist's report before making his examination he is likely to be biased, and the radiologist, aware of the physician's report, is liable to be prejudiced in his interpretation.

Radiologists cannot spend too much time in study of the projected appearances of the normal lungs in people of all ages and occupations; it is only by having a thorough knowledge of the normal that the observer is able to appreciate the abnormal. At the Broken Hill inquiry large numbers of surface workers were examined to obtain an idea of the appearance of the average worker's chest in that district.

\section{Technique}

Fluoroscopic examination should be reduced to a minimum: It is dangerous to the operator, and the information gained by it is of minor importance. It gives no detailed information of lung pathology, but is of value in demonstrating diaphragm movement, in localizing abscess or hydatid, and in the control of lipiodol instillations.

Sufficient attention is not paid by radiolog:sts to the accommodation of the eyes before screening. The operator should be in complete darkness for ten minutes (by the clock) before attempting fluoroscopy. A good rough test of accommodation is the ordinary wrist watch; when the figures appear dazzlingly bright the eyes are accommodated for screening.

In chest radiography the stereoscopic postero-anterior films will give practically all the available information. The lateral film is unnecessary as a routine procedure,

* Read in opening a discussion in the Section of Radiology and Radiotherapeutics at the Annual Meeting of the British Medical Association, Melbourne, 1935. 
but should be used when the postero-anterior films call for it. In young people it is always as well to include an examination of the head sinuses. These stereoscopic films should be viewed in the $x$-ray stereoscope, and not reported on after being viewed as a flat film-a common fault with radiologists.

The stereoscopic films are taken at a distance of six or seven feet, with a four and a half inch vertical tube shift. The central ray for the lower film should be directed between the scapulae in a slightly downward direction. The patient should always be upright, with the hands in front and the shoulders drooped forward ; in this way the scapulae are carried off the chest and good views of the apical regions are obtained. The PotterBucky diaphragm and the Lysholm grid are not recommended owing to the undue exaggeration of fine detail even when films are taken from a distance. The exposure is made at the end of an ordinary inspiration and not at the end of a forced one.

At present, with the exception of the rotalix tube, there is no tube which will economically stand more than 300 milliamperes at 70 kilovolts, and with these factors and using a seven feet distance, it is possible to obtain excellent films of the average working man in $1 / 30$ second. For this work a $20 \mathrm{~kW}$ metallix tube can be used, and the detail obtained, in spite of the coarse focus, is all that can be desired. Violent contrast in chest films is not desirable, and if spinal structure is seen it is a sign of over-penetration.

\section{Apical Scars}

These are dense shadows which appear at the extreme apices. They must not be confused with the shadow of insertion of the sterno-mastoid muscle. They are very common in working men, and are looked upon as evidence of healed tuberculous lesions. They never appear to break down, and if a person with these scars develops tuberculosis it never seems to be associated with this scarred area.

\section{Effusions}

Ordinary fluid collections cause little difficulty in interpretation, but atypical effusions may cause trouble. Interlobar effusions follow the line of the interlobe, and they may assume various shapes. Some of these collections give triangular shadows in the film; sometimes the base is at the periphery, and at other times it is centrally situated. Lateral films are of value in localizing these lesions. Effusions which are mesially situated-that is, between the lung and mediastinum-are rare, and when they occur they are usually associated with an oesophageal lesion. When encountered, a bolus should be administered in a search for an oesophageal condition.

Hydatid shadows are usually spherical, but when situated in the pleural sac they frequently show distortion of outline, with a rounded appearance on the visceral side and a flattened one on the parietal side. An important point in differentiation between ordinary effusions and hydatid is that in effusions there is no depression of the diaphragm, while in hydatid there is marked depression.

\section{Bronchiectasis}

Bronchiectases are troublesome conditions, and they can usually be demonstrated in the stereoscopic films. They can easily be overlooked if situated behind the heart or deep in the costo-phrenic angle.

Lipiodol instillation should only occasionally be called for, and if used it should be introduced by the supraglottic method rather than by injection through the crico-thyroid membrane. With a cocainized pharynx it is very easy to trickle the lip:odol over the epiglottis or to inject it directly into the larynx with a laryngeal syringe. The method of injecting the drug through the crico-thyroid membrane is dangerous, and I have frequently seen cases in which it has been injected into the areolar tissue of the neck, and in one case it had been injected into the oesophagus ; moreover, it is very easy to break a needle against the cartilages.

The amount of lipiodol used should be as small as possible, otherwise it is quite possible to " drown" a portion of the lung. Lipiodol is also a nuisance in after years, as it becomes scattered through the lung and gives appearances similar to tuberculosis or silicosis.

\section{Fibrin Bodies}

Fibrin bodies are seen as rounded bodies on one side of a chest which has been the site of a hydropneumothorax. The fluid coagulates, and this clotted material is formed into rounded ball-like structures. They are difficult to distinguish from secondary malignant deposits, but the latter are generally bilateral. They vary greatly in size, and after the lung becomes fully expanded they gradually disappear and leave no trace.

\section{Fungus Infections}

Fungus infections are rarely met with; the only ones I have seen have been due to yeast infection or to actinomycosis. In the former case there was a bilateral uniform fuzziness without nodule formation or cavitation, but with scattered areas of bronchopneumonic consolidation. Breathlessness was a marked accompaniment. Diagnosis was not made before death.

In actinomycosis the appearances are more definite. The condition is localized to one lobe at first, and it extends in all directions from this original site. It will cross an interlobe, and frequently involves the chest wall, giving a typical boggy oedema of the skin and subcutaneous tissues. It is difficult to differentiate from tuberculosis or malignancy, but in many cases of actinomycosis there is a "vesicular" appearance in the lesion which is quite typical.

\section{Tuberculosis}

Tuberculosis will only receive passing mention. In my experience this disease in the adult practically always starts in the upper lobes. Perihilar and basal tuberculosis is extremely rare. A mottling of the upper half of the chest is practically always tuberculous, while lowerhalf lesions are generally due to catarrhal lesions, abscess, or bronchiectasis.

The early tuberculous lesion is shown as a fluffy mottling in the lung periphery. Fine striae appear about the mottling, due to the formation of fibrous tissue. In acute and miliary tuberculosis this fine mottling increases in distribution and may involve the whole of both lungs, even in the absence of many physical signs. In active disease many of the tuberculous lesions coalesce and may break down in the centre to form cavities. It is not always possible to distinguish between active and quiescent lesions, but an experienced observer becomes very accurate in his interpretation. Lesions with much fibrosis and with clear-cut outlines are generally quiescent.

\section{Silicosis}

The inhalation of large quantities of various dusts over long periods produces changes in the lung fields which may be demonstrated in the film. Such changes occur in rock-choppers, coal miners, cement workers, asbestos workers, and tool grinders. Inhaled dust is dangerous in proportion to its silica content, and the other constituents of the dust are only of incidental importance. 
Most authorities agree that silicates produce no nodular fibrosis in the lungs, but one silicate (that of aluminium and potassium) known as sericite has been put forward as a cause. The general opinion, however, is that it cannot produce these changes by itself, but when present it hastens the action of the silica in producing fibrosis. Leroy Gardner states that sericite will produce inflammation and necrosis but not fibrosis. Asbestos, a silicate of magnesium, produces a diffuse fibrosis and not the nodular fibrosis of silica.

Hawkesbury sandstone contains upwards of 80 per cent. of free slica, metalliferous ores 20 to 60 per cent., while coal carries 2 to 4 per cent. It must be remembered, however, that coal miners are frequently cutting through seams of rock containing 30 per cent. or more of silica, and in many mines sandstone dusting is used to carry down the fine coal dust which is so liable to cause explosions. The analyses of our coal mine dusts as supplied are rather surprising in the small amounts of silica found; from the condition of the lungs of these coal miners a higher percentage of silica would be expected.

Individual susceptibility may play a part in the occurrence of silicos:s ; some men after forty and fifty years of mining in sandstone are frequently unaffected, while others will show changes after six or eight years. With a high silica content in the dust the onset of the disease is more rapid, and in lungs with damaged lymphat:c circulation, whether due to tuberculosis or to chronic bronchitis, the condition develops more rapidly. From other parts of the world cases of silicosis are reported after only a few years' work; this may be due to the greater prevalence of catarrhal conditions with consequent damaged lymphatic circulation and to less healthy conditions of employment abroad.

The extent of the condition as shown in the film cannot be taken as a guide to the worker's disability. Early stages of fibrosis as indicated in the film may be associated with grave disability, while men with a most advanced fibrosis will show no disability.

When silica is inhaled the majority of the particles are arrested in the larger bronchi and expectorated. Only the finer particles (less than 5 microns in diameter) reach the alveoli. The mechanical irritation of dust is not looked upon as of importance, but these particles go into a colloidal solution which acts as tissue irritant producing fibrosis. It is suggested that the silica solution destroys the phagocyte, which stagnates in the lymph stream and by irritation produces the fibrous nodule. Coal particles of similar size are taken up by the phagocyte and carried to the nearest lymph gland area.

The silica nodule is similar to the tuberculous nodule, but it never goes on to caseation. The nodular fibrosis of silicosis is a bilateral condition, and it spreads from the hilum on each side-somewhat more advanced on the right side-and gradually involves the whole of the lung fields. The apices and the lung in the costo-phrenic angles are affected in the very late stages.

In the early stages there is an increase in the shadows of the lung reticulum, but these changes are indistinguishable from the markings in ordinary catarrhal conditions, in asthma, and in the lungs of the aged. At this stage a diagnosis is not possible, but when the nodules are definitely developed there is seen a "stippling " of the lung fields which is character.stic. Of course, there must be changes in the lungs before this stage is reached, but it is not possible, radiographically, to diagnose a " presilicotic " stage. A series of many hundreds of men were tentatively classed as "pre-silicot: " in an investigation carried out by my brother, but the majority failed to develop the typical nodular fibrosis of silicosis.

Once established, silicosis continues to advance, even if the man is removed from his dusty occupation. Pleurisy is a common complication, and extensive adhesions occur to the diaphragm and to the mediastinum, causing distortion of the shadows of the mediastinal contents. The majority of silicotics develop tuberculosis, and when such infection is superadded the condition rapidly approaches a fatal termination. The silicotic lung is a most favourable soil for the tubercle bacillus to flourish in. Coal miners, especially men who have mined in Great Britain, often develop extensive areas of subpleural consolidation of tuberculous origin. This is a very slowly advancing condition, and is probably held in check by the wellknown anti-tuberculous action of coal dust. The appearance of denser areas of mottling in the upper lobes is the sign of superadded tuberculosis; in the silicotic tuberculosis is almost invariably an ap:cal infection.

The differential diagnosis between ordinary tuberculosis and silicosis is generally easy, but it is impossible to make a diagnosis from miliary tuberculosis radiographically. However, the absence of temperature points to a silicosis ; while in tuberculosis there is a tendency for the nodules to coalesce, in silicosis they remain discrete. The fibrosis seen in chronic catarrhal conditions is more a streaking along the bronchial tree than a symmetrically distributed nodular fibrosis.

\section{THE CHILD GUIDANCE CLINIC*}

BY

HARVEY SUTTON, O.B.E., M.D.MELB.

PROFESSOR OF PREVENTIVE MEDICINE AND DIRECTOR, SCHOOL OP PUBLIC HEALTH AND TROPICAL MEDICINE, SYDNEY UNIVERSITY

Problems in behaviour, especially bad habits-the " everyday problems of the everyday child "- have been for many years discussed by parents and others interested in children. Controversy as to school discipline and corporal punishment seems to have originated in England with the appearance of grammar schools of the early sixteenth century. In Elizabethan days schoolmasters showed an implicit belief in the maxim of Solomon, "spare the rod and spoil the child," and the ability to flog pupils became for over two centuries one of the vocational attributes of the pedagogue. Ascham, the tutor of Queen Elizabeth, protested against the universal indiscriminate use of corporal punishment. It is interesting to note the corporal punishment of schoolgirls lately discussed in the British Medical Journal.

John Locke, who, as is sometimes forgotten, was a physician as well as a philosopher and a politician, wrote a book, in every way a modern work, on How to Bring up your Children. The pronoun your is significant, for Locke was a bachelor and exercised the perennial right of the unmarried to instruct parents in their duties. Corporal punishment is shown by him to be a failure, yet its practice was, and to some extent still is, extensively followed. It required a generation of universal (compulsory) education to discredit its use except in a few limited definite breaches of the school rules. Making the punishment fit the crime by making " the prisoner pent-unwillingly represent-a source of innocent merriment" may prove a solution, for ridicule and shame are, as Locke pointed out, powerful influences. Increasing exper:ence, however, points to other methods: just and unemotional criticism, encouragement of good efforts, the selection of sound, interesting outlets for energy, and better personal understanding between teacher and pupil.

* Read in opening a discussion in the Section of Medical Sociology at the Annual Meeting of the British Medical Association at the Annual
Melbourne, 1935. 